\title{
A Numerical Simulation of Scattering from One-Dimensional Inhomogeneous Dielectric Random Surfaces
}

\author{
Kamal Sarabandi, Senior Member, IEEE. Yisok Oh. Student Member, IEEE, and Fawwaz T. Ulaby, Fellow. IEEE
}

\begin{abstract}
In this paper, an efficient numerical solution for the scattering problem of inhomogeneous dielectric rough surfaces is presented. The inhomogeneous dielectric random surface represents a bare soil surface and is considered to be comprised of a large number of randomly positioned dielectric humps of different sizes, shapes, and dielectric constants above an impedance surface. Clods with nonuniform moisture content and rocks are modeled by inhomogeneous dielectric humps and the underiying smooth wet soil surface is modeled by an impedance surface. In this technique, an efficient numerical solution for the constituent dielectric humps over an impedance surface is obtained using Green's function derived by the exact image theory in conjunction with the method of moments. The scattered field from a sample of the rough surface is obtained by summing the scattered fields from all the individual humps of the surface coherently ignoring the effect of multiple scattering between the humps. The statistical behavior of the scattering coefficient $\sigma^{\circ}$ is obtained from the calculation of scattered fields of many different realizations of the surface. Numerical results are presented for several different roughnesses and dielectric constants of the random surfaces. The numerical technique is verified by comparing the numerical solution with the solution based on the small perturbation method and the physical optics model for homogeneous rough surfaces. This technique can be used to study the behavior of scattering coefficient and phase difference statistics of rough soil surfaces for which no analytical solution exists.
\end{abstract}

\section{INTRODUCTION}

I NVESTIGATION of the radar scattering response of natural surfaces is an important problem in remote sensing because of its potential in retrieving desired physical parameters of the surface, namely. its soil moisture content and surface roughness. Soil moisture is a key ingredient of the biochemical cycle and an important variable in hydrology and land processes. Although the problem of electromagnetic wave scattering from random surfaces has been investigated for many years, because of its complexity, theoretical solutions exist only for simple limiting cases. Among the existing theoretical models the small perturbation method (SPM) [1] and the Kirchhoff approximation (KA) [2].can be mentioned, which are applicable for homogeneous surfaces over a restricted regions of validity. Numerous techniques based on the basic assumptions of the SPM and KA have been developed in past in an attempt to improve the region of validity of these

Manuscript received November 15, 1994. This work was supported by NASA under Contract NAGW 2151.

The authors are with the Radiation Laboratory, Department of Electrical Engineering and Computer Science, University of Michigan, Ann Arbor, MI 48109 USA.

Publisher Item Identifier S 0196-2892(96)00996-5. models; however, they all have the basic limitations of the original models [3]. Other theoretical models such as the phase perturbation technique [4] and the integral equation method [5] are not applicable, for inhomogeneous surfaces and their regions of validity have not been fully determined yet. A scattering formulation for rough surfaces with variable surface impedance exist [6]; however, the solution is not applicable to soil surfaces with low dielectric constant. Several numerical solutions of the scattering problem have been proposed to identify the region of validity and accuracy of these theoretical models. Scattering solution for a perfectly conducting random surface using the method of moments has been suggested by Axline and Fung [7]. In this method a tapered incident field is used as the excitation to eliminate the edge effect contribution.

A numerical solution for homogeneous dielectric random surfaces has been reported [8] where again a tapered illumination is used to limit the size of the scatterer. The accuracy of the numerical solution with tapered illumination decreases with increasing incidence angle. To our knowledge, numerical scattering solution for inhomogeneous rough surfaces does not exist.

Analysis of microwave backscatter observations by $\mathrm{Oh}$ et al. [9] reveals that the existing theoretical models cannot adequately explain the scattering behavior of soil surfaces. The deviation between theoretical predictions and experimental data is attributed to three factors. First, the roughness parameters are often outside the region of validity of the theoretical models. Second, the autocorrelation functions measured from natural surfaces are very complicated and are not Gaussian or exponential correlation functions. Finally, the most important reason is that the natural surfaces are not homogeneous in most cases, i.e., the moisture content is not uniform in depth. The top rough layer which includes clods and rocks is usually dry and the underlying soil layer is moist and smooth.

In this paper, we model a soil surface as an inhomogeneous dielectric random surface comprised of a large number of randomly positioned two-dimensional dielectric humps of different sizes, shapes, and dielectric constants all lying above an impedance surface. At microwave frequencies, the moist and smooth underlying soil layer can be modeled as an impedance surface, and the irregularities above it can be treated as dielectric humps of different dielectric constants and shapes. For the field scattered by a single dielectric hump over an impedance surface, we have an available efficient numerical solution that uses the exact image theory for the 
Green's function in conjunction with the method of moments [10]. In the solution of a single hump, it has been shown that the bistatic scattered field is very weak at points in close proximity to the impedance surface; thus, the effects of multiple scattering between humps can be ignored. In this case, the scattered field from a collection of randomly positioned dielectric humps can easily be obtained by summing the scattered field of all the constituent humps coherently. The scattering coefficients $\left(\sigma^{\circ}\right)$ is obtained by a Monte Carlo simulation.

In Section II, we summarize the procedure for the numerical solution of a single hump above an impedance surface. Section III outlines the procedures used for generating the random surfaces and for evaluating the statistics of the scattered field. Numerical results and their comparison with theoretical models are presented in Section IV.

\section{SCATTERING FROM INDIVIDUAL HUMPS}

In this section, we briefly review the procedure for the numerical solution of scattering from a two-dimensional dielectric object above a uniform impedance surface [10]. The radiated field for a dipole source above a dissipative halfspace medium (Green's function) is usually evaluated using the Sommerfeld integral [11]. This infinite integral, in general, is highly oscillatory and computationally rather inefficient. In [10], the Green's function of an impedance surface was derived in terms of rapidly converging integrals using appropriate integral transforms. The scattering problem was then formulated by integral equations which were solved numerically using the method of moments.

Suppose a dielectric object, possibly inhomogeneous, is located above an impedance surface and is illuminated by a plane wave. The incident field $\boldsymbol{E}^{\boldsymbol{i}}$ induces conduction and displacement currents in the dielectric object which together are known as the polarization current $J_{e}$. The polarization current can be represented in terms of the total electric field inside the dielectric object, which is comprised of the incident, reflected, and scattered fields denoted by $E^{i}, E^{r}$, and $E^{s}$, respectively. Thus

$$
J_{e}(\bar{\rho})=-i k_{0} Y_{0}[\epsilon(\bar{\rho})-1]\left[E^{i}(\bar{\rho})+E^{r}(\bar{\rho})+E^{s}(\bar{\rho})\right]
$$

where $k_{0}=\omega \sqrt{\mu_{0} \epsilon_{0}}, Y_{0}=\sqrt{\epsilon_{0} / \mu_{0}}$, and $\epsilon(\bar{\rho})$ is the relative dielectric constant of the object at the point $\bar{\rho}=x \hat{x}+y \hat{y}$. The fields $E^{i}, E^{r}$, and $E^{s}$ are, respectively, given by

$$
\begin{aligned}
& \boldsymbol{E}^{i}(\bar{\rho})=\left(E_{h}^{i} \hat{h}_{i}+E_{v}^{i} \hat{v}_{i}\right) \exp \left[i k_{0} \hat{k}_{i} \cdot \bar{\rho}\right] \\
& \boldsymbol{E}^{r}(\bar{\rho})=\left(R_{h} E_{h}^{i} \hat{h}_{r}+R_{v} E_{v}^{i} \hat{v}_{r}\right) \exp \left[i k_{0} \hat{k}_{r} \cdot \bar{\rho}\right] \\
& \boldsymbol{E}^{s}(\bar{\rho})=i k_{0} Z_{0} \int_{S} \overline{\bar{G}}\left(\bar{\rho}, \bar{\rho}^{\prime}\right) \cdot J_{e}\left(\bar{\rho}^{\prime}\right) d \bar{\rho}^{\prime}
\end{aligned}
$$

where $E_{h}^{i}$ and $E_{v}^{i}$ are the horizontal (E-polarized) and vertical (H-polarized) components of the incident field, respectively. $R_{h}$ and $R_{v}$ are the horizontal and vertical Fresnel reflection coefficients and $\overline{\bar{G}}\left(\bar{\rho}, \bar{\rho}^{\prime}\right)$ is the dyadic Green's function of the problem.

There is no known exact solution for the integral equation given by (1). Hence, an approximate numerical solution of this equation must be obtained using the method of moments.
This is done by dividing the cross section of the dielectric structure into $N_{c}$ sufficiently small rectangular cells such that the dielectric constant and the polarization current over each cell can be approximated by constant values. Using the pointmatching technique, the integral equation can be cast into a matrix equation of the following form:

$$
\left[\begin{array}{ccc}
{\left[\mathcal{Z}_{x x}\right]} & {\left[\mathcal{Z}_{x y}\right]} & 0 \\
{\left[\mathcal{Z}_{y x}\right]} & {\left[\mathcal{Z}_{y y}\right]} & 0 \\
0 & 0 & {\left[\mathcal{Z}_{z z}\right]}
\end{array}\right]\left[\begin{array}{l}
{\left[\mathcal{I}_{x}\right]} \\
{\left[\mathcal{I}_{y}\right]} \\
{\left[\mathcal{I}_{z}\right]}
\end{array}\right]=\left[\begin{array}{l}
{\left[\mathcal{V}_{x}\right]} \\
{\left[\mathcal{V}_{y}\right]} \\
{\left[\mathcal{V}_{z}\right]}
\end{array}\right]
$$

where $\left[\mathcal{Z}_{p q}\right]$ is the impedance matrix, $\left[\mathcal{I}_{p}\right]$ is the unknown vector whose entries are the values of the polarization current at the center of each cell, and $\left[\mathcal{V}_{p}\right]$ is the excitation vector with $p=x, y$, or $z$. The entries of $\left[\mathcal{V}_{p}\right]$ are simply given by

$$
\begin{aligned}
v_{p, n}= & i k_{0} Y_{0}\left[\epsilon\left(x_{n}, y_{n}\right)-1\right] \\
& \cdot\left\{\left[E_{p}^{i}\left(x_{n}, y_{n}\right)+E_{p}^{r}\left(x_{n}, y_{n}\right)\right]\right\} \cdot \hat{p}
\end{aligned}
$$

and the entries of $\left[\mathcal{Z}_{p q}\right]$ can be evaluated from

$$
\begin{aligned}
z_{p q m n}= & -\delta_{p q} \delta_{m n}+k_{0}^{2}\left[\epsilon\left(x_{n}, y_{n}\right)-1\right] \\
& \cdot \int_{\Delta S_{m}}\left[G_{p q}\left(x_{n}, y_{n} ; x_{m}, y_{m}\right)\right. \\
& \left.+G_{r p q}\left(x_{n}, y_{n} ; x_{m}, y_{m}\right)\right] d s_{m}
\end{aligned}
$$

where $\delta_{p q}$ and $\delta_{m n}$ are the Kronecker delta functions, and $p, q=x, y$, or $z$.

Explicit expressions for the elements of the impedance matrix are given in [10] where off-diagonal elements are obtained by approximating the Green's function via its Taylor series expansion around the midpoint of each cell and then the integration over the cell surface is performed analytically. For diagonal elements the free-space Green's function is approximated by its small argument expansion and then integration is performed analytically.

\section{Monte Carlo Simulation OF ROUGH SURFACE SCATTERING}

The Monte Carlo scattering simulation of rough surfaces made up of a finite collection of dielectric humps involves in five major steps as shown in Fig. 1. The first step is to choose the type (size, shape, and dielectric constant, etc.) and number of constituent humps. The second step deals with generating a surface sample by positioning a large number of humps with a prescribed probability. The third step in this algorithm is to compute the inverse impedance matrices for all constituent humps using the numerical method explained in the previous section. Next the scattered field from the surface is computed by coherent summation of the scattered fields from all of the humps in the surface sample. Finally the scattering coefficient $\sigma^{\circ}$ is obtained by repeating the fourth step for a large number of independently generated surface samples.

The types of constituent humps which in addition to their probability of occurrence fully characterize the statistics of a random surface. Fig. 2 shows the geometry and dielectric profiles of different types of dielectric humps which can be handled by this algorithm. For example, Fig. 2(a) shows a typical hump for a dry clod above a moist and smooth underlying soil layer $\left(\epsilon_{0}<\epsilon_{1}<\epsilon_{2}\right)$ and Fig. 2(b) shows the same 


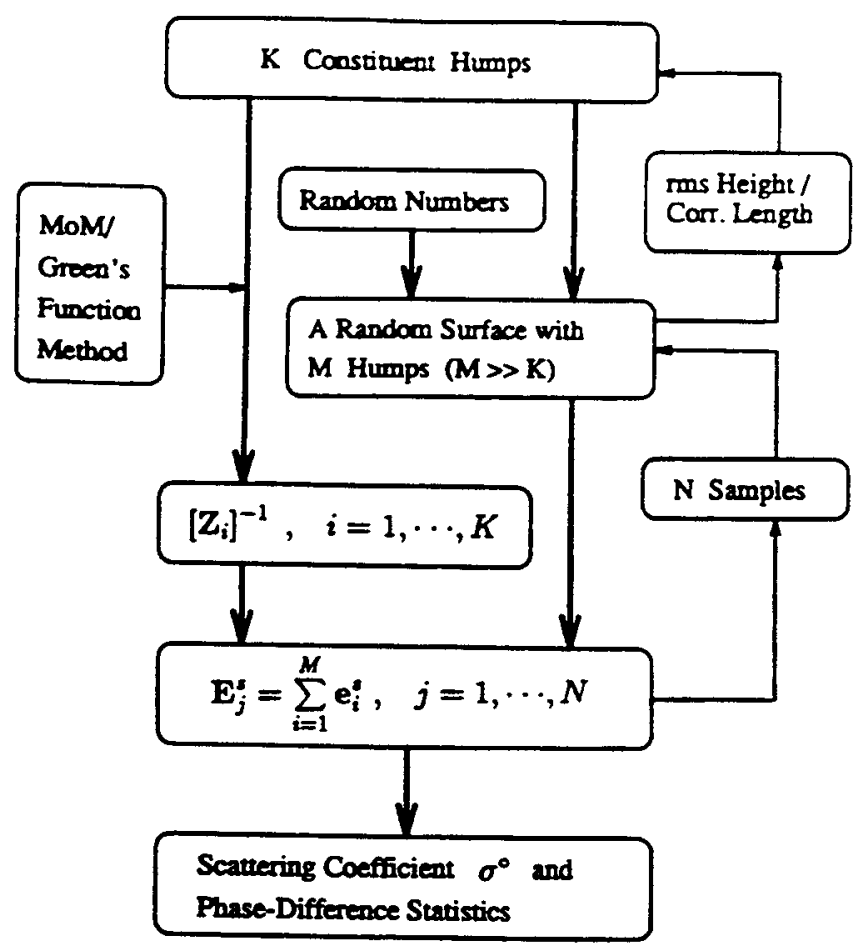

Fig. 1. Flow char of the Monte Carlo simulation for the rough surface problem.

hump when the clod and underlying layer are both moist (a homogeneous surface). The hump itself may be considered to be inhomogeneous as shown in Fig. 2(c). Isolated irregularities such as rocks above a flat surface can be represented by the hump shown in Fig. 2(d) where the bump occupies only a part of the total width allocated for an individual hump. When the surface is rough with a short correlation length, the geometry of the humps are more complicated and two examples are shown in Fig. 2(e) and (f). The profiles of Fig. 2(a)-(e) used in this paper are given by the following functional forms

$$
\begin{aligned}
y(x)= & \frac{W}{A} \cos ^{2}\left(\frac{\pi x}{W}\right), \quad-\frac{W}{2} \leq x \leq \frac{W}{2}, \\
& \text { for (a)-(c) } \\
y(x)= & A\left(1-\frac{x^{2}}{B^{2}}\right), \quad-B \leq x \leq B, B \leq W, \\
& \text { for (d) }
\end{aligned}
$$

and

$$
y(x)=A F_{1}(x)+B F_{2}(x)
$$

with

$$
\left.\begin{array}{rl}
F_{1}(x) & =\cos ^{n}\left(\frac{\pi x}{W}-\frac{\pi}{2}\right)\left(\frac{x}{W}\right)^{m} \\
F_{2}(x) & =\cos ^{n}\left(\frac{\pi x}{W}-\frac{\pi}{2}\right)\left(1-\frac{x}{W}\right)^{m}
\end{array}\right\}
$$

where $A$ and $B$ are constants, $n$ and $m$ are integers, and $W$ is width of a hump. The set of constituent humps for a surface can be constructed by choosing a finite number of parameters in the desired functionals and the desired dielectric constants. The profile of Fig. 2(f) is very complicated and should be obtained

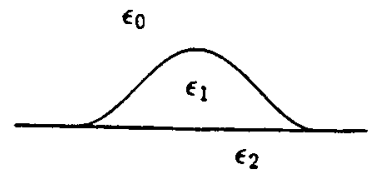

(a)

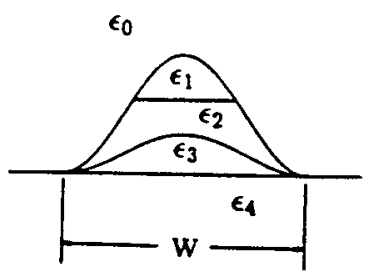

(c)

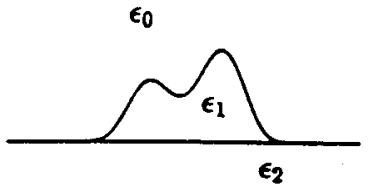

(e)

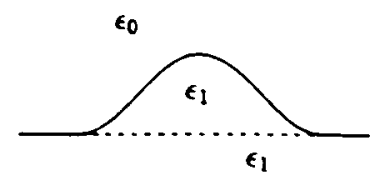

(b)

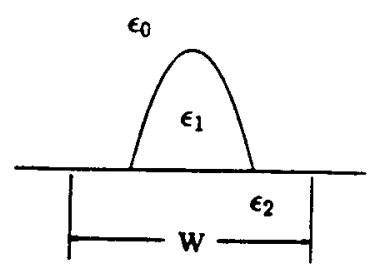

(d)

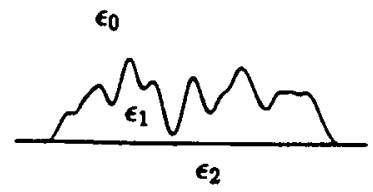

(f)
Fig. 2. Hump types considered in this paper.

numerically by the procedure outlined in [8]. In this procedure the hump profile is obtained from a sequence of independent Gaussian deviates with zero mean and unit variance which are correlated by a set of weighting factors derived from the desired correlation function.

Suppose the set of individual humps includes $K$ different humps (including size, shape, and dielectric constant) and the profiles of the humps in the set are represented by $f_{i}(x)$, $i=1, \cdots, K$. Then a sequence of random numbers ranging from 1 to $K$, which is generated by a random number generator with the prescribed probability distribution, is used to position a large number of humps randomly to construct a surface sample. If the total number of humps $(M)$ in the surface sample is much larger than the number of constituent humps $(K)$ and the random number generator has a uniform distribution, the probability of the occurrence of each hump in the surface will be about $M / K$. A functional form of the generated surface profile can be represented by

$$
y(x)=\sum_{m=1}^{M} f_{i_{m}}\left(x-\sum_{l=1}^{m-1} W_{i_{l}}\right)
$$

where $i_{m}, i_{l} \in\{1, \cdots, K\}$ and $W_{i_{l}}$ represents the width of the hump of the $i_{l}$ th type. The roughness parameters, rms height $s$, correlation length $l$, and rms slope $m$, can be computed either numerically or analytically from the surface profile given in (11). However, the analytical computation is only possible for simple functional forms and simple probability distribution functions. Assuming perfect randomness, the average height of the surface can be computed from

$$
\bar{y}(x)=\frac{1}{L} \sum_{i=1}^{K} p_{i} \int_{0}^{W_{i}} f_{i}(x) d x
$$

where $L=\sum_{i=1}^{K} p_{i} W_{i}$ and $p_{i}$ is the probability of occurrence of the hump of $i$ th type. The $\mathrm{ms}$ height $s$ and the rms slope 
$m$, respectively, can be evaluated from

$$
\begin{aligned}
s & =\left\langle[y(x)-\bar{y}(x)]^{2}\right\rangle^{1 / 2} \\
& =\left\{\frac{1}{L} \sum_{i=1}^{K} \int_{0}^{w_{i}}\left[p_{i} f_{i}(x)-\bar{y}(x)\right]^{2} d x\right\}^{1 / 2}
\end{aligned}
$$

and

$$
\begin{aligned}
m & =\left\langle\left[\frac{d y(x)}{d x}-\left\langle\frac{d y(x)}{d x}\right\rangle\right]^{2}\right\rangle^{1 / 2} \\
& =\left\{\frac{1}{L} \sum_{i=1}^{K} w_{i} \int_{0}^{W_{i}}\left[f_{i}^{\prime}(x)\right]^{2} d x\right\}^{1 / 2} .
\end{aligned}
$$

Assuming the surface has a Gaussian correlation function, the correlation length $l$ in terms of $\mathrm{mm}$ height and rms slope is given by

$$
l=\sqrt{2} \frac{s(\mathrm{~ms} \text { height })}{m \text { (rms slope })}
$$

It is often required to generate a random surface of given rms height $s$ and correlation length $l$. In that case the required surface can be obtained by an iterative process where some initial values for the hump parameters are chosen. Then the roughness parameters are calculated and compared with the desired ones. Depending on the difference between the calculated $s$ and $l$ and the desired $s$ and $l$ the hump parameters are modified and this process is repeated until the difference is below a tolerable error.

Once the set of individual humps for a random surface with given $s$ and $l$ is formed the impedance matrices, $\left[\mathcal{Z}_{p q}\right]_{i}$, $i=1, \cdots, K$, can be computed using the method of moments described in the previous section. Since the scattered field of a hump near the impedance surface is very weak the effect of multiple interaction between humps in a surface sample can be ignored. Therefore, by inverting and storing the impedance matrices of the constituent humps the scattered field of any surface sample comprised of $M$ humps $(M \gg$ $K$ ) and for any incidence and observation directions can be computed very efficiently. For a given direction of incidence the polarization current in the $j$ th hump for the vertical and horizontal polarization, respectively, are given by

$$
\left[\begin{array}{l}
{\left[\mathcal{I}_{x}\right]} \\
{\left[\mathcal{I}_{y}\right]}
\end{array}\right]_{j}=\left[\begin{array}{ll}
{\left[\mathcal{Z}_{x x}\right]} & {\left[\mathcal{Z}_{x y}\right]} \\
{\left[\mathcal{Z}_{y x}\right]} & {\left[\mathcal{Z}_{y y}\right]}
\end{array}\right]_{i_{j}}^{-1}\left[\begin{array}{l}
{\left[\mathcal{V}_{x}\right]} \\
{\left[\mathcal{V}_{y}\right]}
\end{array}\right]_{j}
$$

and

$$
\left[\mathcal{I}_{z}\right]_{j}=\left[\mathcal{Z}_{z z}\right]_{i_{j}}^{-1}\left[\mathcal{V}_{z}\right]_{j}
$$

where $j \in\{1, \cdots, M\}$ and $i_{j} \in\{1, \cdots, K\}$ representing the hump of the $i$ th type. The excitation vector $[\mathcal{V}]_{j}$ is computed from (6) where the position vector $\bar{\rho}$ is specified by the discretization procedure and the profile function (11). The electric polarization current induced inside the surface sample can be represented by

$$
\begin{gathered}
{\left[\mathcal{J}_{p}\right]=\left[\left[\mathcal{I}_{p}\right]_{1}^{T}, \cdots,\left[\mathcal{I}_{p}\right]_{i}^{T}, \cdots,\left[\mathcal{I}_{p}\right]_{M}^{T}\right]^{T}} \\
p=x, y, z
\end{gathered}
$$
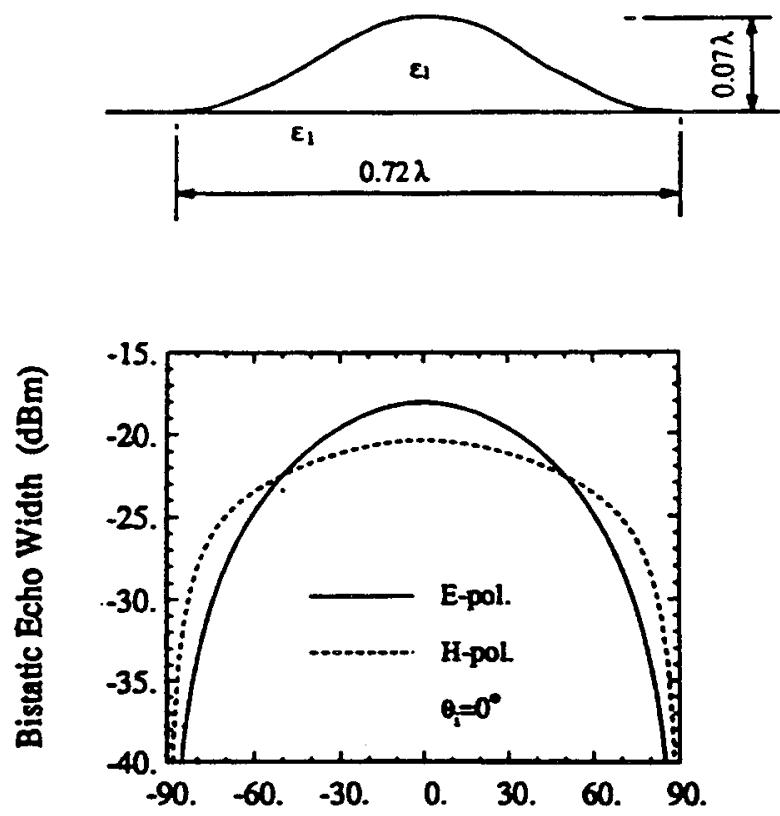

Scatter Angle (Degrees)

(a)

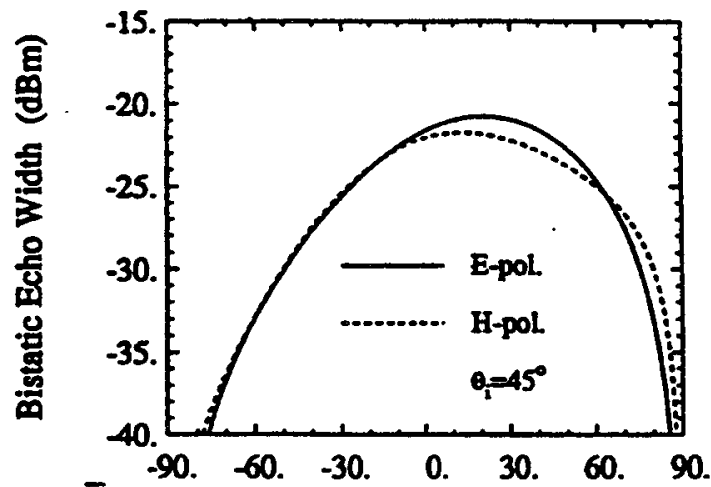

Scatter Angle (Degrees)

(b)

Fig. 3. Bistatic echo width of a squand-cosine hump of $\epsilon_{1}=15+i 3$, $W=0.72 \lambda, H=0.07 \lambda$ over an impedance surface of $\eta=0.254-i 0.025$ at (a) $\theta_{i}=0^{\circ}$ and (b) $\theta_{i}=45^{\circ}$ at $f=5 \mathrm{CHz}$ for E- and H-polarizations.

where $\left[\mathcal{I}_{p}\right]_{i}$ is the $p$-polarized current inside the $i$ th hump. The radiated far field can be evaluated from

$$
E_{p p}^{s}=\sqrt{\frac{2}{\pi k_{0} \rho}} e^{i\left(k_{0} \rho-\dot{\pi} / 4\right)} S_{p p}, \quad p p=h h \text { or } v v
$$

where $S_{p p}$ is the far field amplitude given by

$$
\begin{aligned}
S_{h h}= & -\frac{k_{0} Z_{0}}{4} \sum_{n=1}^{N_{t}} J_{z}\left(x_{n}, y_{n}\right) \Delta x_{n} \Delta y_{n} e^{-i k_{0} \sin \theta_{s} x_{n}} \\
& \cdot\left[e^{-i k_{0} \cos \theta_{c} y_{n}}+R_{E}\left(\theta_{s}\right) e^{i k_{0} \cos \theta_{s} y_{n}}\right] \\
S_{v v}= & \frac{k_{0} Z_{0}}{4} \sum_{n=1}^{N_{t}} \Delta x_{n} \Delta y_{n} e^{-i k_{0} \sin \theta_{a} x_{n}} \\
& \cdot\left\{J_{x}\left(x_{n}, y_{n}\right) \cos \theta_{s}\right. \\
& \cdot\left(\left[e^{-i k_{0} \cos \theta_{0} y_{n}}-R_{H}\left(\theta_{s}\right) e^{i k_{0} \cos \theta_{a} y_{n}}\right)\right.
\end{aligned}
$$




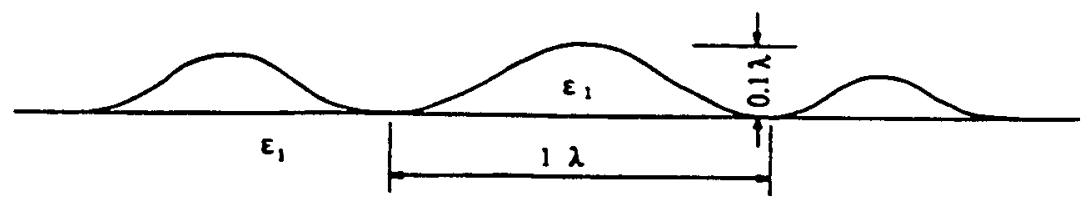

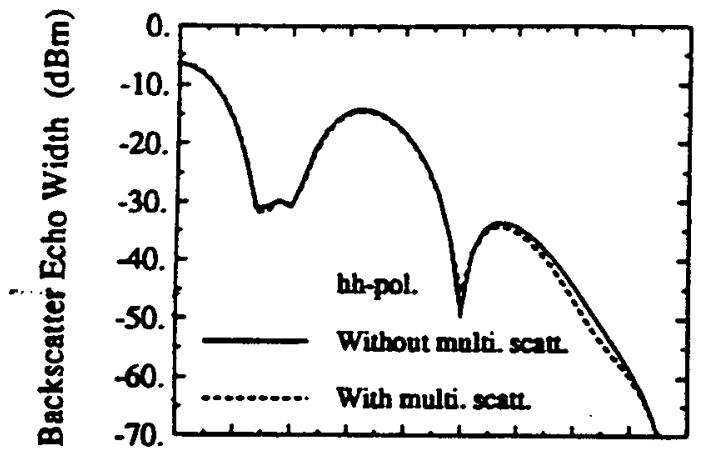

0. 10. 20. 30. 40. 50. 60. 70. 80.90 .

Incidence Angle (Degrees)

(a)

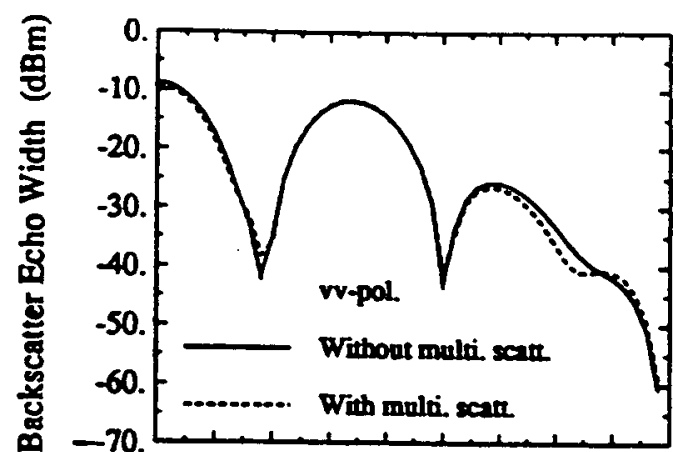

0. 10. 20. 30. 40. 50. 60. 70. 80.90 .

Incidence Angle (Degrees)

(b)

Fig. 4. Multiple scattering effect on the backscatter echo width of a surface segment consisting of three squared-cosine humps with $\epsilon_{1}=15+i 3$ at $f=5 \mathrm{GHz}$ for both (a) HH-polarization and (b) VV-polarization.

$$
\begin{aligned}
& -J_{y}\left(x_{n}, y_{n}\right) \sin \theta_{s} \\
& \left.\cdot\left(e^{-i k_{0} \cos \theta_{s} y_{n}}+R_{H}\left(\theta_{s}\right) e^{i k_{0} \cos \theta_{s} y_{n}}\right)\right\} .
\end{aligned}
$$

Here $N_{t}$ is the total number of cell in the surface sample.

The statistical behavior of the scattered field are obtained from the scattered fields $E_{p p}^{s}$ of many independent surface samples. For sufficiently large $N_{s}$ surface samples the incoherent scattering coefficient is computed from

$$
\begin{gathered}
\sigma_{p p}^{\circ}=\lim _{\rho \rightarrow \infty} \frac{2 \pi \rho}{N_{s} L_{a v}}\left[\sum_{j=1}^{N_{s}}\left|E_{p p, j}^{s}\right|^{2}-\frac{1}{N_{s}}\left|\sum_{j=1}^{N_{s}} E_{p p, j}^{s}\right|^{2}\right] \\
p p=h h, v v
\end{gathered}
$$

where $L_{a v}=\left(1 / N_{s}\right) \sum_{j=1}^{N_{s}} L_{j}$, and $L_{j}$ is the total length of $j$ th random surface.

\section{NUMERICAL RESULtS}

To demonstrate the performance of the technique proposed in this paper, we shall use it to compute the scattering for some sample surfaces and then compare the results with those predicted by the available theoretical scattering models, when conditions apply. First, we consider a surface with homogeneous dielectric humps as shown in Fig. 2(a). The functional form of the humps are given by (8) where the parameters $A$ and $W$ are varied to generate the set of the constituent humps. Keeping $A$ as a constant controlling the height and varying $W$, a set of similar humps can be generated. A random number generator with output $i \in\{1, \cdots, K\}$ selects the parameter $W_{i}=B \lambda i$, where $B$ is a constant controlling the width of the humps and $\lambda$ is the wavelength. In this example, the hump parameters were chosen according to Table I and the random number generator was given a uniform distribution with $K=10$. Before presenting the statistical scattering behavior of the surface, it is useful to demonstrate the validity of the assumption regarding the significance of the effects of multiple scattering among the humps. Fig. 3(a) and (b) show the bistatic echo width of a squared-cosine hump with $W=0.72 \lambda, H=0.07 \lambda, \epsilon_{1}=15+i 3$ above a surface with $\eta=0.254-i 0.025$ (which corresponds to $\epsilon_{2}=15+i 3$ ) at $5 \mathrm{GHz}$ when the incidence angle $\theta_{i}=0^{\circ}$ and $\theta_{i}=45^{\circ}$, respectively. It is shown that the bistatic echo widths at the large scatter angles (near the surface) are very weak which implies that the effect of multiple scattering between humps can be ignored. In order to illustrate the effect of multiple scattering, a surface segment consisting of three squared-cosine humps with $\epsilon_{1}=15+i 3$ above an impedance surface with $\eta=0.254-i 0.025$ was considered (see Fig. 4). Dimensions of three humps are, respectively, given by: $W_{1}=0.8 \lambda, H_{1}=0.08 \lambda ; W_{2}=1.0 \lambda, H_{2}=0.1 \lambda$, and $W_{3}=0.6 \lambda, H_{3}=0.06 \lambda$. The backscatter echo widths of the surface segment were computed twice. In one case the scattered field was computed from the polarization current of isolated humps (ignoring the effect of mutual coupling) and in the other case the polarization current of the threehump structure was obtained directly from the method of moments solution (including the effect of mutual coupling). Fig. 4(a) and (b) show that the effect of multiple scattering is negligible for both polarizations. As long as the ratio of rms height to correlation length of the surface $(s / l)$ is small, this approximation provides accurate results. For most natural surfaces $s / l<0.3$ which satisfies this condition [9]. However, if the ratio $(s / l)$ is relatively large, the hump type of Fig. 2(f) must be used to include the effect of multiple scattering at the expense of computation time.

The rms surface height $s$ and the rms surface slope $m$ for this surface can be computed from (13) and (14), respectively, 
TABLE I

Rolghness Parnmeters Correspondeng to Constants A aNd $B$

\begin{tabular}{|c|c|c|c|c|c|c|c|c|c|}
\hline \multirow[b]{2}{*}{ Case } & \multirow[b]{2}{*}{$A$} & \multirow[b]{2}{*}{$B$} & \multicolumn{2}{|c|}{ Approx. $\dagger$} & \multicolumn{2}{|c|}{ Exact $\ddagger$} & \multicolumn{2}{|c|}{ At $5 \mathrm{GHz}$} & \multirow[b]{2}{*}{ Remarks } \\
\hline & & & $\begin{array}{l}s \\
\text { in }\end{array}$ & $\begin{array}{l}l \\
m\end{array}$ & $\begin{array}{l}s \\
\text { in }\end{array}$ & $\begin{array}{l}l \\
m\end{array}$ & ks & $k l$ & \\
\hline 1 & 30 & 0.20 & 0.115 & 2.21 & 0.115 & 2.03 & 0.12 & 2.13 & SPM region \\
\hline 2 & 30 & 0.36 & 0.208 & 3.98 & 0.207 & 3.63 & 0.22 & 3.80 & \\
\hline 3 & 30 & 0.70 & 0.405 & 7.74 & 0.405 & 7.15 & 0.42 & 7.49 & PO region \\
\hline
\end{tabular}

$\dagger$ Approximation by equations (23), (24) and (15).

$\ddagger$ Numerical evaluation with $\mathbf{4 0 0 0}$ humps,

$s$ : rms surface height,

$l$ : correlation length.

TABLE II

Constants Used in the Numerical Computations

\begin{tabular}{c|c|c|c|c|c|c|c}
\hline & \multicolumn{2}{|c|}{ Individual hump size } & \multicolumn{2}{c|}{ No. of humps } & Length of & No. of \\
Case & \multicolumn{2}{|c|}{ Width } & \multicolumn{2}{c}{ Height } & for each & surface & segments \\
No. & min. & max. & min. & max. & surface & segment & for a \\
& $(\lambda)$ & $(\lambda)$ & $(\lambda)$ & $(\lambda)$ & segment & $(\lambda)$ & surface \\
\hline 1 & 0.2 & 2.0 & 0.0066 & 0.066 & 40 & 44 & 100 \\
2 & 0.36 & 3.6 & 0.012 & 0.12 & 40 & 79 & 100 \\
3 & 0.7 & 7.0 & 0.023 & 0.233 & 40 & 154 & 100 \\
\hline
\end{tabular}

and are given by

$$
\begin{aligned}
s & =\left[\frac{1}{L_{W}} \sum_{i=1}^{K}\left(\frac{3}{8} \frac{W_{i}^{3}}{A^{2}}-\frac{W_{i}^{2}}{A} \bar{y}+W_{i} \bar{y}^{2}\right)\right]^{1 / 2} \\
m & =\frac{\pi}{\sqrt{2} A}
\end{aligned}
$$

where

and

$$
\bar{y}=\frac{1}{2 A L_{W}} \sum_{i=1}^{K} W_{i}^{2}
$$

$$
L_{W}=\sum_{i=1}^{K} W_{i} .
$$

It should be noted that the rms surface slope $m$ of this surface depends only on the constant $A$. Therefore, for a fixed value of $A$, both the rms height and the correlation length increase at the same rate with increasing $B$. Table I shows several values of roughness parameters, $s$ and $l$, corresponding to different values of $A$ and $B$.

A random number generator was used to select and position 4000 squared-cosine humps over the impedance surface ( $\eta=$ $0.254-i 0.025)$. Then this surface was divided into 100 segments to obtain 100 independent surface samples each having 40 humps. The length of the surface segment was chosen to be about $44 \lambda$ to $154 \lambda$ depending on the correlation length of the surface, corresponding to the size of individual humps. Table II summarizes the characteristics of the surfaces and their constituent humps used in the examples considered in this study.

The backscattering coefficients for the surface at $5 \mathrm{GHz}$ with $k s=0.12$ and $k l=2.13$ (Case 1 in Tables $I$ and II) are computed by the Monte Carlo simulation technique for 


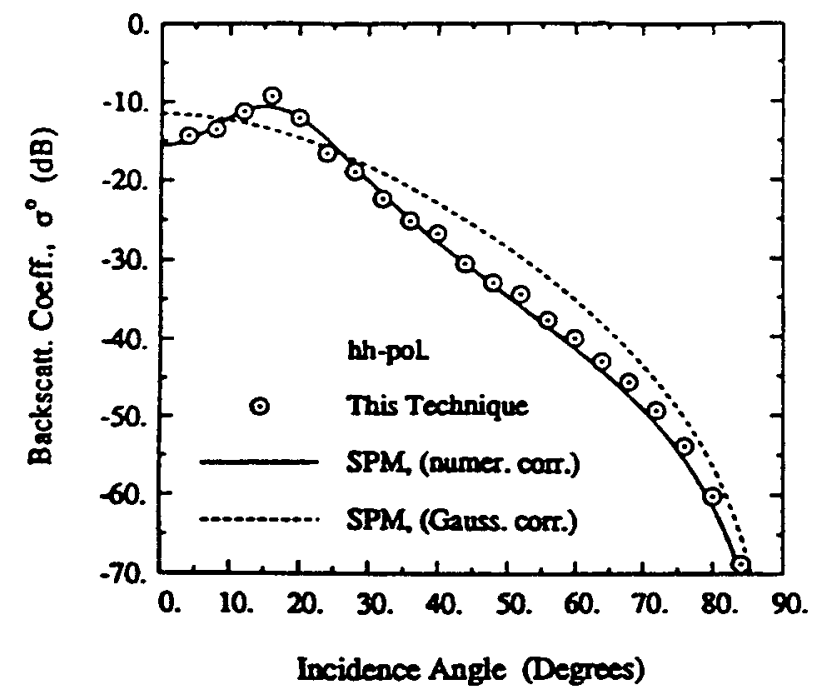

(a)

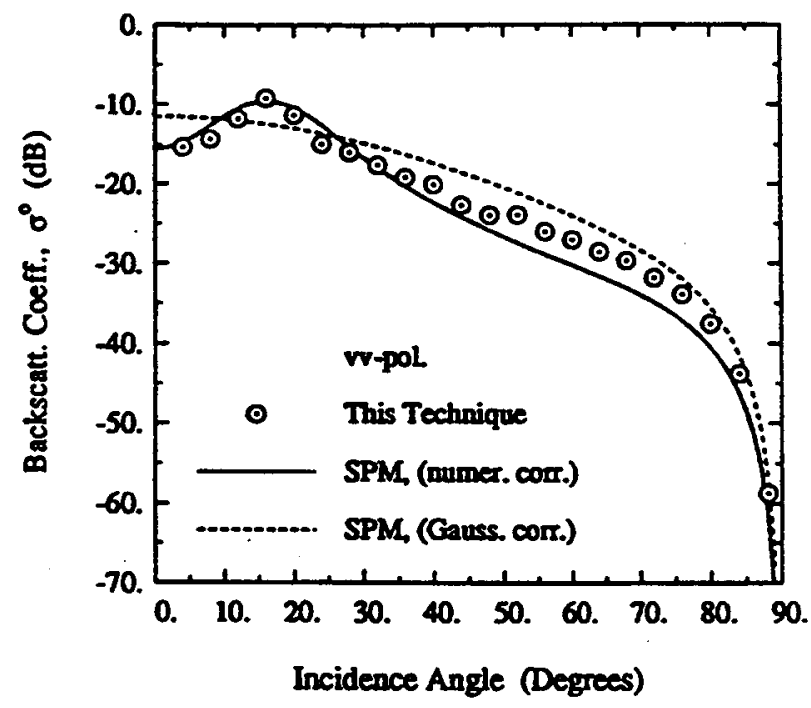

(b)

Fig. 5. Backscattering coefficient $\sigma^{\circ}$ of the random surface with $k s=0.12, k l=2.13$, and $\epsilon_{1}=\epsilon_{2}=15+i 3$ as computed by the SPM and the numerical technique. (a) HH-polarization. (b) VV-polarization.

a homogeneous surface with $\epsilon_{1}=\epsilon_{2}=15+i 3$ (Fig. 2(b)), and compared with the analytical results based on the SPM. The comparisons are shown in Fig. 5(a) and (b). For the SPM solution, the scattering coefficient $\sigma^{\circ}$ is proportional to the roughness spectrum (Fourier transform of the correlation function). Both the actual and Gaussian correlation functions are used in the calculation of the backscattering coefficients using the SPM. It is shown that the Monte Carlo simulation agrees very well with the SPM prediction when the actual correlation function is used. The discrepancies between the Monte Carlo simulation and the SPM with Gaussian correlation function indicate the importance of the tail section of the correlation function in the estimation of $\sigma^{\circ}$.

The numerical simulation was also performed for a surface at $5 \mathrm{GHz}$ with $k s=0.42, k l=7.49$ (Case 3 in Tables I and II), and $\epsilon_{1}=\epsilon_{2}=15+i 3$. The roughness parameters of this surface fall within the validity region of the physical

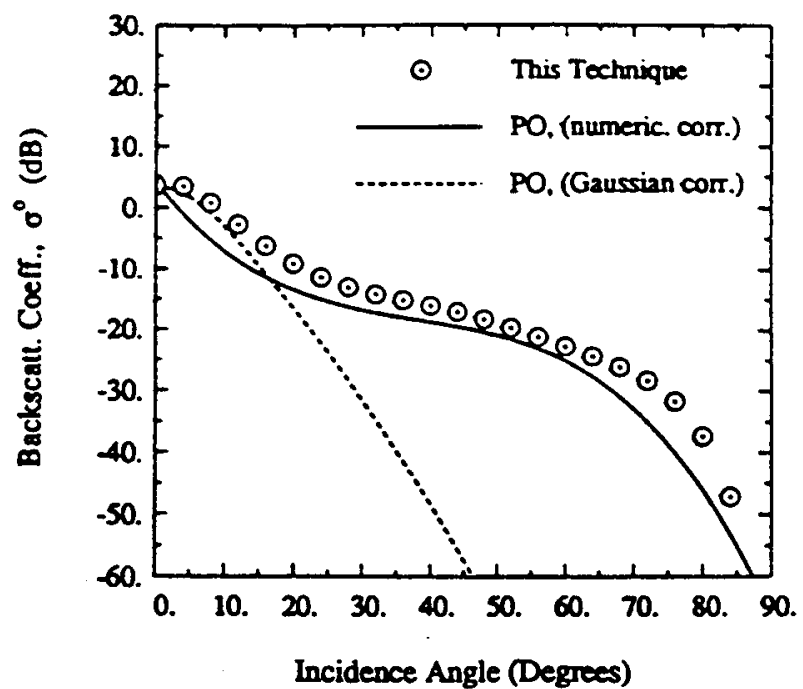

Fig. 6. Backscancring coefficient $\sigma^{\circ}$ of the random surface with $k s=0.42, k l=7.49$, and $\epsilon_{1}=\epsilon_{2}=15+i 3$ as computed by the PO model and the numerical technique for HH-polarization.

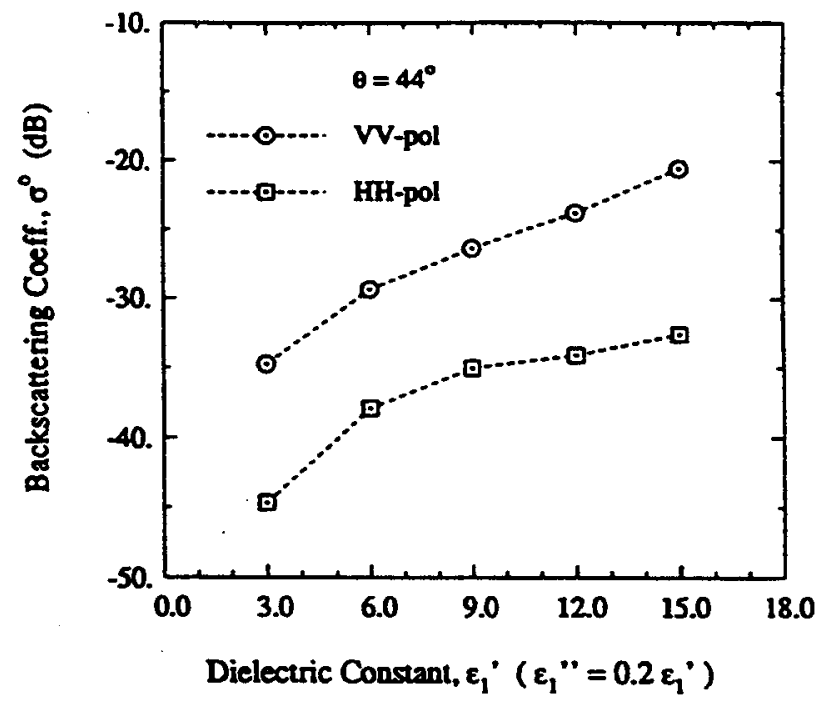

Fig. 7. The sensitivity of the backscattering coefficient $\sigma^{\circ}$ to the dielectric constant in case of $k s=0.22, k l=3.8$, and $\epsilon_{2}=15+i 3$ at $\theta=44^{\circ}$.

optics (PO) model; therefore, the numerical solution can be compared with the PO solution. The scattering coefficient $\sigma_{h h}^{\circ}$ predicted by the $\mathrm{PO}$ model using the actual correlation function agrees very well with the results computed by the numerical technique (see Fig. 6). In this figure the PO solution using a Gaussian correlation function with the same correlation length as the actual comrelation function is also compared with the numerical simulation. It is shown that the agreement is good only for low incidence angles $\left(\theta_{i} \leq 20^{\circ}\right)$ and the discrepancy between the two solutions becomes rather significant for higher incidence angles. In this case, similar to the previous case (SPM), it is shown that the tail of the correlation function plays an important role in determining the angular patterns of the backscattering coefficients.

With the success of the Monte Carlo simulation in predicting the scattering behavior of rough surfaces in the small perturba- 
tion and physical optics regions. the numerical model can be used to study complex surfaces with intermediate roughness parameters and inhomogeneous dielectric profiles. For example. the sensitivity of radar backscatter to the moisture content of the top layer for the Case 2 random surface is shown in Fig. 7 at $\theta=44^{\circ}$.

\section{CONCLUSION}

In this paper an efficient Monte Carlo simulation technique is proposed for computing electromagnetic scattering by inhomogeneous one-dimensional rough surfaces. The surface irregularities are represented by inhomogeneous dielectric humps of different shapes and the underlying layer is represented by an impedance surface. A moment-method procedure, in conjunction with the exact image theory, is used for calculation of the field scattered by the dielectric humps. It was shown that the scattered field near the impedance surface is weak, and hence, the effect of multiple scattering between humps can be ignored.

To check the validity of the Monte Carlo simulation, the numerical results were compared with the existing analytical solutions for surfaces at extreme roughness conditions. A smooth surface that satisfies the validity region of the SPM and a surface that satisfies the validity region of the PO model were considered, and in both cases excellent agreements were obtained between the analytical results and those computed using the proposed technique. It was found that away from normal incidence, the tail of the correlation function plays an important role in the determination of the backscattering coefficients.

The analysis presented in this paper is only for onedimensional surfaces and therefore is incapable of predicting the cross-polarized scattering coefficients. A numerical simulation for a two-dimensional rough surface using a similar method is computationally tractable.

\section{REFERENCES}

[1] S. O. Rice, "Reflection of electromagnetic waves by slightly rough surfaces," Commun. Pure Appl. Math., vol. 4, pp. 351-378, 1951.

[2] P. Beckmann and A. Spizzichino, The Scattering of Electromagnetic Waves from Rough Surfaces. Norwood, MA: Artech House, 1987.

[3] G. S. Brown, "Backscattering from a Gaussian distributed perfectly conducting rough surface," IEEE Trans. Antennas Propagat., vol. AP-26, pp. 472-482, May 1978.

[4] D. Wineberner and A. Ishimaru, "Investigation of a surface field phase perturbation technique for scattering from rough surfaces," Radio Sci., vol. 20, pp. 161-170, Mar. 1985.

[5] A. K. Fung and G. W. Pan, "A scattering model for perfectly conducting random surfaces: I. Model development and II. Range of validity," Int. J. Remote Sensing, vol. 8, no. 11, pp. 1579-1605, 1987.

[6] E. Bahar, "Radio wave propagation over a rough variable impedance boundary: Part I. Full-wave analysis," IEEE Trans. Antennas Propagat., vol. AP-20, pp. 354-362, May 1972.
[7] R. M. Axtine ani A. K. Fung. "Numencal computation of scattenns from a perfectly conducung random surface." IEEE Trans Antennas Propagal., vol. AP.26. pp. 482-48S. Ma! 1978.

181 A.K. Fung and M. F. Chen. "Numencal simulation of seatterng from simple and composite random surfaces," J. Opt. Soc. Amer. A vol. 2. no. 12. pp. 2274-2284. Dec. 1985.

(9) Y. Oh. K. Sarabandi. and F. T. Ulaby. "An empirical model and an inversion technique for radar scattering from bare soil surfaces." IEEE Trans. Geosci. Remose Sensing, vol. 30. pp. 370-382. M1ar. 1992

[10] K. Sarabandi, "Scattering from dielectric structures above impedance surfaces and resistive sheets," IEEE Trans. Antennes Propagal.. vol. to. pp. 67-78, Jan. 1992

[11] J. A. Stratton, Electromagnetic Theon: New York: McGraw-Hill, 1941.

[12] A. K. Fung and M. F. Chen. "Numerical simulation of scattering from simple and composite random surfaces," J. Opt. Soc. Amer.. vol. 2. no. 12. pp. 2274-2284. Dec. 1985.

[13] F. T. Ulaby, M. K. Moore, and A. K. Fung, Microwove Remote Sensing, Active and Passive, vol. 2 and 3. Norwood, MA: Artech House. 1986.

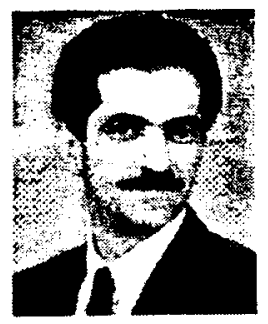

Kamal Sarabandi (S'87-M'90-SM'93) received the B.S. degree in electrical engineering from Sharif University of Technology, Tehran. Iran. in 1980. He entered the graduate program at the University of Michigan in 1984 and received the M.S.E. degree in electrical engineering in 1986, and the M.S. degree in mathernatics and the Ph.D. degree in electrical engineering in 1989.

From 1980-1984, he was a Microwave Engineer with the Telecommunication Research Center in Iran. He is presently an Assistant Professor in the Department of Electrical Engineering and Computer Science at the University of Michigan. He has 15 years of experience with microwave sensors and radar systems. In the past six years, he has served as Principal Investigator and CoInvestigator on many projects sponsored by NASA, JPL, ARO, DARPA, etc., all related in one way or the other to the radar remote sensing of environment. He has published four book chapters and more than fifty papers in refereed joumals on electromagnetic scattering, random media modeling, microwave measurement techniques, radar calibration, application of neural networks in inverse scattering problems, and microwave sensors. He has also had more than 80 papers and presentations in national and international conferences and symposia on similar subjects.

Dr. Sarabandi is listed in Who's Who in Electromagnetics and is the Chaiman of Geoscience and Remote Sensing Society Southeastern Michigan chapter.

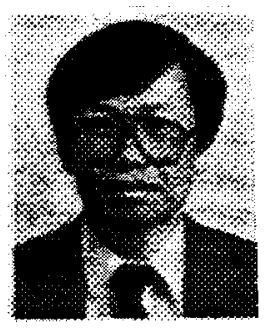

Yisok Oh (S'88) received the B.S. degree from Yonsei University, Seoul, Korea, in 1982, the M.S. degree from the University of Missouri, Rolla, in 1988 , and the $P h_{1} D$. degree from the University of Michigan, Ann Artor, in 1993, all in electrical engineering.

He is presently an Assistant Professor in the Department of Radio Engineering at the Hong IK University. His research interests include electromagnetic wave scattering from random surfaces and microwave remote sensing.

Fawwaz T. Ulaby (M'68-SM'74-F'80), for a photograph and biography, see p. 99 of the January 1996 issue of this TRANSACTIONS. 